\title{
Release-Rebound Processes: Vector Motions
}

\author{
Roman TEISSEYRE \\ Institute of Geophysics, Polish Academy of Sciences, Warszawa, Poland \\ e-mail: rt@igf.edu.pl
}

\begin{abstract}
The tensor relations describing the shear deviatoric strains and rotation strains may be presented as vector relations in a special coordinate system, e.g., in the diagonal or off-diagonal one. However, these fields can be also presented in the 4D invariant forms by means of invariant Dirac tensors. We present 4D relativistic relations for the invariant shear deviatoric strain and rotation strain vectors closely related to a fracture process in solids and to the molecular strains (shear and rotational) in fluids. These shear and rotation strains may interact with the radial derivatives of pressure along the propagation directions.
\end{abstract}

Key words: release-rebound, Dirac tensors, strain vectors, fracture relations.

\section{INTRODUCTION}

The release-rebound motions originate due to the fracture processes; to present such relations we will follow the 4D Maxwell space-time system. We assume that at a source of elastic waves, the molecular transport motions become transformed to the real fracture motion as a source of the observed radiated fields, that is, the rotation and shear strains.

\section{RELEASE-REBOUND PROCESSES; VECTOR MOTIONS}

In our considerations on some important relations for vectors and tensors in a special coordinate system, e.g., in the diagonal or off-diagonal one, we can demand that any of such relations may be presented in an invariant 4D form.

Ownership: Institute of Geophysics, Polish Academy of Sciences;

(C) 2015 Teisseyre. This is an open access article distributed under the Creative Commons Attribution-NonCommercial-NoDerivs license, http://creativecommons.org/licenses/by-nc-nd/3.0/. 
Thus, we underline that such important relations, which in the 3D space may be achieved only in a special coordinate system, could become invariant in the 4D system, quite similarly to the electro-magnetic fields; we may call these relations the 4D Maxwell-like invariant relations.

For the total symmetric and antisymmetric strains, the axial, shear, and rotation strains, $\bar{E}, \hat{E}_{(i k)}, \breve{E}_{[i k]}$, can be written as:

$$
\begin{array}{ll}
E_{i k}=E_{(i k)}+E_{[i k]}=\delta_{i k} \bar{E}+\hat{E}_{(i k)}+\breve{E}_{[i k]} & \text { - total strains, } \\
\bar{E}(\bar{U})=\frac{1}{3} \sum_{s} E_{s s}=\frac{1}{3} \sum_{s} \frac{\partial \bar{U}_{s}}{\partial x_{s}} & \text { - axial strain, } \\
\hat{E}_{(i k)}(\hat{U})=E_{(i k)}-\delta_{i k} \bar{E}=\frac{1}{2}\left(\frac{\partial \hat{U}_{k}}{\partial x_{i}}+\frac{\partial \hat{U}_{i}}{\partial x_{k}}\right)-\delta_{i k} \frac{1}{3} \sum_{s=1}^{3} \frac{\partial \bar{U}_{s}}{\partial x_{s}} & \text { - shear deviatoric strains, } \\
\breve{E}_{[i k]}(\breve{U})=\frac{1}{2}\left(\frac{\partial \breve{U}_{k}}{\partial x_{i}}-\frac{\partial \breve{U}_{i}}{\partial x_{k}}\right) & \text { - rotation strain, }
\end{array}
$$

where we have used the potential displacement fields, $\bar{U}_{s}, \hat{U}_{k}, \breve{U}_{n}$, which could be treated as quite independent potentials.

We write also for pressure related terms:

$$
\bar{E}=1 / 3 \sum E_{s s}, \quad \bar{S}=1 / 3 \sum S_{s s}, \quad \bar{S}=(2 \mu+3 \lambda) \bar{E}, \quad \text { and } p=-\bar{S},
$$

where the axial strain relates to pressure.

However, the symmetric, shear strains could be presented in the special off-diagonal system also as a vector field defined in that system; thus, we obtain:

$$
\begin{aligned}
& E_{(i)} \rightarrow\left\{E_{(23)}, E_{(31)}, E_{(12)}\right\}, \quad E_{[i]} \rightarrow\left\{E_{[23]}, E_{[31]}, E_{[12]}\right\}, \\
& E_{[s]}=\frac{1}{2} \varepsilon_{s k l} E_{[k l]}, \quad E_{(s)}=\frac{1}{2}\left|\varepsilon_{s k l}\right| E_{(k l)}^{D}, \quad E_{i}=E_{[i]}+i E_{(i)} .
\end{aligned}
$$

However, we may present these strains as the complex invariant vectors; to this end, we should define the special off-diagonal vectors (that means, the vectors defined in the off-diagonal system). To achieve this goal we should define the invariant complex rotation and shear strain vectors by using the Dirac tensors in the system $\{\mathbf{x}$, ict $\}$ : 


$$
\begin{aligned}
& \gamma^{1}=\left[\begin{array}{cccc}
0 & 0 & 0 & -1 \\
0 & 0 & 1 & 0 \\
0 & 1 & 0 & 0 \\
-1 & 0 & 0 & 0
\end{array}\right], \quad \gamma^{2}=\left[\begin{array}{cccc}
0 & 0 & -1 & 0 \\
0 & 0 & 0 & -1 \\
-1 & 0 & 0 & 0 \\
0 & -1 & 0 & 0
\end{array}\right] \\
& \gamma^{3}=\mathrm{i}\left[\begin{array}{cccc}
0 & 0 & 0 & 1 \\
0 & 0 & 1 & 0 \\
0 & -1 & 0 & 0 \\
-1 & 0 & 0 & 0
\end{array}\right], \quad \gamma^{4}=\mathrm{i}\left[\begin{array}{cccc}
1 & 0 & 0 & 0 \\
0 & 1 & 0 & 0 \\
0 & 0 & -1 & 0 \\
0 & 0 & 0 & -1
\end{array}\right] \text {, } \\
& \gamma^{1} \gamma^{3}=\left[\begin{array}{cccc}
i & 0 & 0 & 0 \\
0 & -i & 0 & 0 \\
0 & 0 & i & 0 \\
0 & 0 & 0 & -i
\end{array}\right], \quad \gamma^{2} \gamma^{3}=\left[\begin{array}{cccc}
0 & i & 0 & 0 \\
i & 0 & 0 & 0 \\
0 & 0 & 0 & -i \\
0 & 0 & -i & 0
\end{array}\right], \quad \gamma^{1} \gamma^{2}=\left[\begin{array}{cccc}
0 & 1 & 0 & 0 \\
-1 & 0 & 0 & 0 \\
0 & 0 & 0 & -1 \\
0 & 0 & 1 & 0
\end{array}\right], \\
& \gamma^{1} \gamma^{4}=\left[\begin{array}{cccc}
0 & 0 & 0 & i \\
0 & 0 & -i & 0 \\
0 & i & 0 & 0 \\
-i & 0 & 0 & 0
\end{array}\right], \quad \gamma^{2} \gamma^{4}=\left[\begin{array}{cccc}
0 & 0 & i & 0 \\
0 & 0 & 0 & i \\
-i & 0 & 0 & 0 \\
0 & -i & 0 & 0
\end{array}\right], \quad \gamma^{3} \gamma^{4}=-\left[\begin{array}{cccc}
0 & 0 & 0 & 1 \\
0 & 0 & 1 & 0 \\
0 & 1 & 0 & 0 \\
1 & 0 & 0 & 0
\end{array}\right]
\end{aligned}
$$

and

$$
\gamma^{4} \gamma^{2} \gamma^{3}=\left[\begin{array}{cccc}
0 & -1 & 0 & 0 \\
-1 & 0 & 0 & 0 \\
0 & 0 & 0 & -1 \\
0 & 0 & -1 & 0
\end{array}\right]
$$

Now, for the vector $E_{s}=E_{[s]}+i E_{(s)} \rightarrow E_{\sigma}$ we may write

$$
E_{\lambda \kappa}=E_{[\lambda \kappa]}+i E_{(\lambda \kappa)} \rightarrow E_{23} \gamma^{1}+E_{31} \gamma^{2}+E_{12} \gamma^{4} \gamma^{2} \gamma^{3}=\left[\begin{array}{cccc}
0 & -E_{3} & -E_{2} & -E_{1} \\
-E_{3} & 0 & E_{1} & -E_{2} \\
-E_{2} & E_{1} & 0 & -E_{3} \\
-E_{1} & -E_{2} & -E_{3} & 0
\end{array}\right]
$$

or equivalently for $E_{23} \equiv E_{1}, E_{31} \equiv E_{2}, E_{12} \equiv E_{3}$ :

$$
E_{\sigma}=E_{[\sigma]}+i E_{(\sigma)}=E_{1} \gamma^{1}+E_{2} \gamma^{2}+E_{3} \gamma^{4} \gamma^{2} \gamma^{3}=\left[\begin{array}{cccc}
0 & -E_{3} & -E_{2} & -E_{1} \\
-E_{3} & 0 & E_{1} & -E_{2} \\
-E_{2} & E_{1} & 0 & -E_{3} \\
-E_{1} & -E_{2} & -E_{3} & 0
\end{array}\right] \text {. }
$$


Thus, the wave equations for deviatoric shear and rotation strains could be presented in the following way:

$$
\sum_{n} \frac{\partial^{2}}{\partial x_{n}^{2}} E_{\lambda n}-\frac{\kappa^{2} \partial^{2}}{c^{2} \partial t^{2}} E_{\lambda n}=Y_{\lambda n}, \quad \text { or } \quad \square E_{\lambda \eta}=Y_{\lambda \eta},
$$

where instead of the related strain wave velocity we introduce the material constant, $\kappa$, and the invariant light velocity $c,(V=c / \kappa)$.

We may add the external forces, $Y_{\lambda k}$ :

$$
Y_{\lambda \kappa}=\mathrm{i} Y_{23} \gamma^{1}+\mathrm{i} Y_{31} \gamma^{2}+Y_{12} \gamma^{3}=\left[\begin{array}{cccc}
0 & Y_{12} & -Y_{31} & -Y_{23} \\
-Y_{12} & 0 & Y_{23} & -Y_{31} \\
Y_{31} & -Y_{23} & 0 & -Y_{12} \\
Y_{23} & Y_{31} & Y_{12} & 0
\end{array}\right],
$$

or

$$
Y_{\mu}=\mathrm{i} Y_{1} \gamma^{1}+\mathrm{i} Y_{2} \gamma^{2}+Y_{3} \gamma^{3}=\left[\begin{array}{cccc}
0 & Y_{3} & -Y_{2} & -Y_{1} \\
-Y_{3} & 0 & Y_{1} & -Y_{2} \\
Y_{2} & -Y_{1} & 0 & -Y_{3} \\
Y_{1} & Y_{2} & Y_{3} & 0
\end{array}\right] \text {. }
$$

In this way we arrive at the equations for the deviatoric shear and rotation strain vectors, $E_{s}=E_{[s]}+i E_{(s)} \rightarrow E_{\sigma}$ :

$$
\sum_{n} \frac{\partial^{2}}{\partial x_{n}^{2}} E_{\sigma}-\frac{\kappa^{2} \partial^{2}}{c^{2} \partial t^{2}} E_{\sigma}=Y_{\sigma}, \quad \text { or } \quad \square E_{\sigma}=Y_{\sigma} .
$$

We assume that these complex relations could be split into the following Maxwell-like equations for the vectors $E_{(s)}, E_{[i]}$ :

$$
\varepsilon_{s p q} \frac{\partial}{\partial x_{p}} E_{[q]}-\frac{\kappa}{c} \frac{\partial}{\partial t} E_{(s)}=\frac{4 \pi \kappa}{c} J_{s}, \quad \varepsilon_{s p q} \frac{\partial}{\partial x_{p}} E_{(q)}+\frac{\kappa}{c} \frac{\partial}{\partial t} E_{[s]}=0,
$$

where $\partial E_{[s]} / \partial x_{s}=0, \partial E_{(s)} / \partial x_{s}=4 \pi \varepsilon$, and we have introduced the defect related current field, $J_{k}$, and propagation velocity $V=c / \kappa$, and the big constant, $\kappa$, depends on a given material and related conditions.

The Maxwell-like relations can describe both the strain wave propagation and also the fracture events occurring in different conditions and materials.

The related 4D form for these Maxwell-like relations (Eq. 7a) can be presented as follows:

$$
\sum_{\kappa} \frac{\partial}{\partial x^{\kappa}} E_{\lambda \kappa}=\frac{4 \pi \kappa}{c} J_{\lambda}, \quad x^{\lambda}=\left\{x^{1}, x^{2}, x^{3}, x^{4}\right\}, \quad x^{4}=\mathrm{i} \frac{c}{\kappa} t .
$$


At a fracture process, e.g., an earthquake, a wave propagation will be excited (cf., Teisseyre 1985, 2011). The related wave mosaic explains the interrelated propagation of shear and rotation motions.

We may note that a compressional load might induce defects forming the centers with opposite-sense shears and rotations, and, accordingly, the micro-breaks may lead to the fragmentation processes (rebound slip). We should note that the theories of dislocation and dislocation arrays (Eshelby et al. 1951, cf., Rybicki 1986) lead to a better understanding of the roles of the defect densities and applied stresses (cf., Kossecka and DeWitt 1977 and Teisseyre and Górski 2012) which brings us closer to our understanding of the fracture processes and dynamic synchronization.

Note that the Gibbs free energy of defect formation has been recently specified by Majewski and Teisseyre (2013) by succesfully extending a thermodynamical model; for a recent review see Varotsos (2007).

We should underline that the propagation of shear and rotation strain waves presents the mutual interaction of shears and rotation strains; such a linear propagation is visualized in Fig. 1.

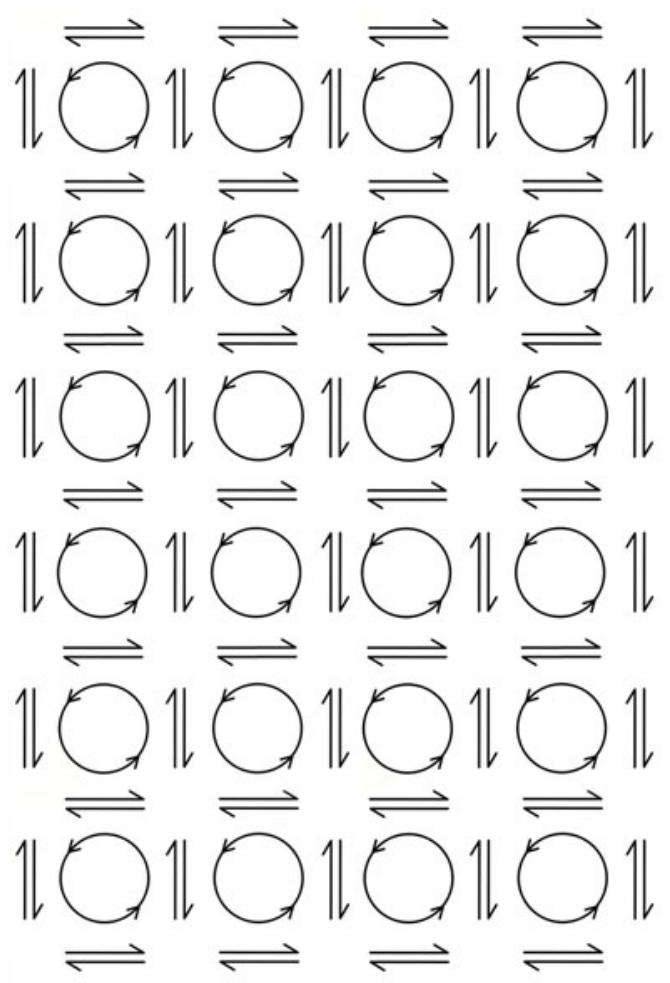

Fig. 1. Propagation of the shear and rotation strains, a linear case. 


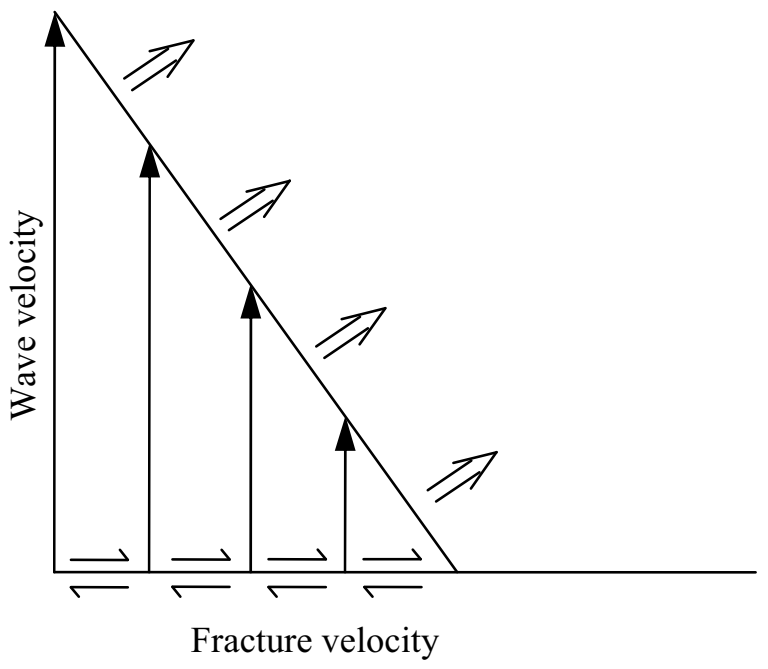

Fig. 2. Sketch showing how the fracture velocity and wave velocity form the propagation front velocity (double arrows) in the near-fracture region.

In a near-fracture region we can assume that a fracture progress at the source induces the waves propagating much faster than the fracture velocity; in this way, a propagation velocity front becomes created (Fig. 2). Thus, a fracture progress and waves propagation lead together to the front propagation; its velocity depends on the velocities of both, fracture and wave. This remark relates to the problems of the fault-plane solutions.

Finally, we should come to the fracture-related equations based on the release-rebound processes applied to the molecular motions: the molecular displacements and simple molecular rotation. The radiated rotation and shear strains can influence, however, any local molecular transport behaviour.

In relation to the real propagation of the shear and rotation strain waves, we may present a behaviour of the molecular transport; its propagation is a consequence of the propagation of shear and rotation strain waves. In this sense, we may define the molecular displacement transport, $w_{s}=\partial v_{s} / \partial x_{s}$.

In this way we may consider the molecular motions appearing in the plane $z=$ const and thus we may consider the 2D case with motions on plane $(r, \varphi)$. The related molecular transport motions, along the propagation plane, relate to the $(r, \varphi)$ system:

$$
w_{n} \rightarrow\left(w_{r}, w_{\varphi}\right) .
$$


In this case we have assumed $w_{z}=0$. The rotation transport molecular vector, $\vec{\omega}_{s}$, may interact with these fields. Thus, to this local molecular transport components we add the molecular rotation transport with the component, $\vec{\omega}_{z}$, oriented along the $z$-direction:

$$
\vec{\omega}_{s} \rightarrow \vec{\omega}_{z} \quad \text { and } \quad\left(\vec{\omega}_{r}=0, \vec{\omega}_{\varphi}=0\right) .
$$

This molecular shear transport and molecular rotation transport should be subjected to local release-rebound fracture equations ( $c f$., Eq. 7a):

$$
\varepsilon_{s p q} \frac{\partial}{\partial x_{p}} \vec{\omega}_{q}-\frac{\tilde{\kappa} \partial}{c \partial t} w_{s}=0, \quad \varepsilon_{s p q} \frac{\partial}{\partial x_{p}} w_{q}+\frac{\tilde{\kappa} \partial}{c \partial t} \vec{\omega}_{s}=0 .
$$

To assure the 4D invariance we have put, instead of the molecular velocity, $v$, the invariant velocity, $c$, and some material constant, $\tilde{\kappa}: v=c / \tilde{\kappa}$.

We include here the molecular rotation transport (Eq. 8b) and in this way the Eqs. 9a become invariant.

For the considered case, related to the molecular motions, we obtain from Eqs. 9a:

$$
\frac{\partial \vec{\omega}_{Z}}{r \partial \varphi}-\frac{\partial w_{r}}{v \partial t}=0, \quad \frac{\partial \vec{\omega}_{Z}}{\partial r}+\frac{\partial w_{\varphi}}{v \partial t}=0, \quad \frac{\partial w_{\varphi}}{\partial r}-\frac{\partial \vec{\omega}_{Z}}{v \partial t}=0 .
$$

or

$$
\frac{\partial}{\partial t} \frac{\partial \vec{\omega}_{Z}}{r \partial \varphi}-\frac{\partial^{2} w_{r}}{\nu \partial t^{2}}=0, \quad \frac{\partial}{\partial t} \frac{\partial \vec{\omega}_{Z}}{\partial r}+\frac{\partial^{2} w_{\varphi}}{\nu \partial t^{2}}=0, \quad \frac{\partial}{\partial t} \frac{\partial w_{\varphi}}{\partial r}-\frac{\partial^{2} \vec{\omega}_{Z}}{\nu \partial t^{2}}=0 .
$$

This, quite new system of relations for $w_{r}, w_{\varphi}$, and $\vec{\omega}_{Z}$, leads to the equation for the molecular rotation:

$$
\frac{\partial^{2} \vec{\omega}_{Z}}{\partial r^{2}}+\frac{\partial^{2} \vec{\omega}_{z}}{v^{2} \partial t^{2}}=0
$$

The related source fracture processes may occur with some phase delay, e.g., $v=i v_{0}$; we obtain:

$$
\frac{\partial^{2} \vec{\omega}_{Z}}{\partial r^{2}}-\frac{\partial^{2} \vec{\omega}_{Z}}{v_{0}^{2} \partial t^{2}}=0
$$

Similarly we may consider also the radial wave propagation (cf., Fig. 3). In this case, we should consider the local molecular system $(r, \varphi)$ perpendicular to the $R$ radial direction; for the related local shear and rotation strains we may add the presented transport: 


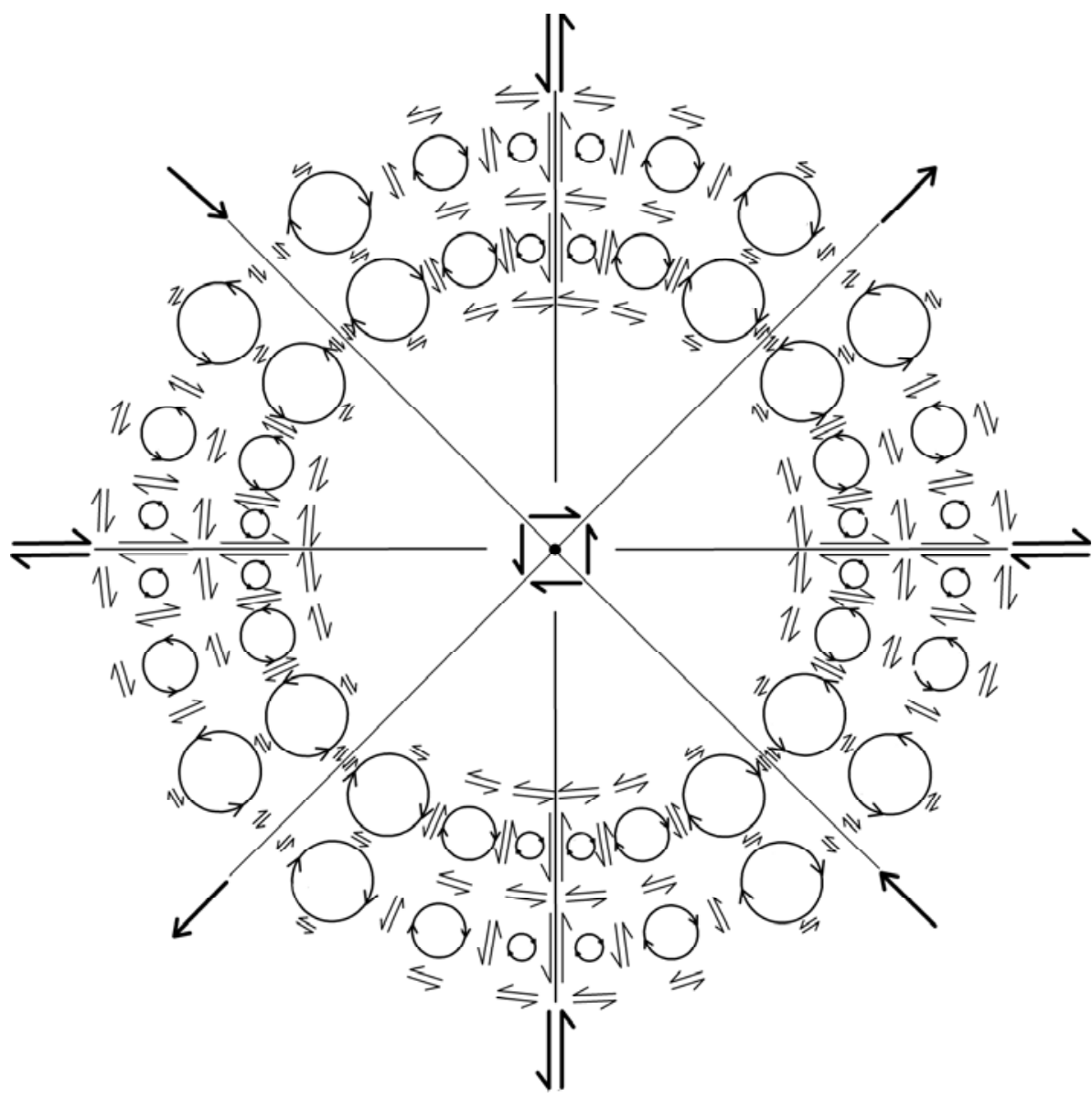

Fig. 3. Radial wave propagation for the shear and rotation strains (the rotation strains decrease near the shear axes to zero while the shear strains decrease between those axes).

$$
w_{n} \rightarrow\left(w_{\varphi}, w_{r}\right)
$$

The molecular radial rotation component, $\vec{\omega}_{z}$, and these molecular transport components, $w_{r}$ and $w_{\varphi}$, should be subjected to release-rebound fracture equations with specific velocity-like term $v(c f$., Eqs. 9a)

$$
\varepsilon_{s p q} \frac{\partial}{\partial x_{p}} \vec{\omega}_{q}-\frac{\partial}{v \partial t} w_{s}=0, \quad \varepsilon_{s p q} \frac{\partial}{\partial x_{p}} w_{q}+\frac{\partial}{v \partial t} \vec{\omega}_{s}=0,
$$

where 


$$
\begin{aligned}
& \varepsilon_{s p q} \frac{\partial}{\partial x_{p}} \vec{\omega}_{q} \rightarrow\left\{0, \frac{\partial \vec{\omega}_{R}}{\partial z},-\frac{\partial \vec{\omega}_{R}}{R \partial \varphi}\right\} \text { at } \vec{\omega}_{\varphi}=0, \vec{\omega}_{z}=0, \\
& \varepsilon_{s p q} \frac{\partial}{\partial x_{p}} w_{q} \rightarrow\left\{\frac{\partial w_{z}}{R \partial \varphi}-\frac{\partial w_{\varphi}}{\partial z},-\frac{\partial w_{z}}{\partial R}, \frac{\partial w_{\varphi}}{\partial R}\right\} \text { at } w_{R}=0 .
\end{aligned}
$$

We obtain three relations for $w_{\varphi}, w_{z}$, and $\vec{\omega}_{R}$ :

$$
\frac{\partial \vec{\omega}_{R}}{\partial z}-\frac{\partial w_{\varphi}}{v \partial t}=0, \quad \frac{\partial \vec{\omega}_{R}}{r \partial \varphi}+\frac{\partial w_{z}}{v \partial t}=0
$$

and

$$
\frac{\partial w_{z}}{R \partial \varphi}-\frac{\partial w_{\varphi}}{\partial z}+\frac{\partial \vec{\omega}_{R}}{v \partial t}=0, \quad \text { for a given } R \text { value }
$$

We have obtained finally a quite new system of relations for $w_{\varphi}, w_{z}$, and $\vec{\omega}_{R}$, with a special velocity-like term $v$, eliminating from these relations the terms related to molecular rotations we obtain the following equations for the molecular displacements:

$$
\frac{\partial^{2} w_{\varphi}}{\partial z^{2}}-\frac{\partial^{2} w_{z}}{R \partial \varphi \partial z}-\frac{\partial^{2} w_{\varphi}}{v^{2} \partial t^{2}}=0, \quad \frac{\partial^{2} w_{z}}{R^{2} \partial \varphi^{2}}-\frac{\partial^{2} w_{\varphi}}{R \partial \varphi \partial z}-\frac{\partial^{2} w_{z}}{v^{2} \partial t^{2}}=0,
$$

and for the molecular rotation (molecular vortex structure):

$$
\frac{\partial^{2} \vec{\omega}_{R}}{R^{2} \partial \varphi^{2}}+\frac{\partial^{2} \vec{\omega}_{R}}{\partial z^{2}}-\frac{\partial^{2} \vec{\omega}_{R}}{v^{2} \partial t^{2}}=0
$$

These last Eqs. 13a, b are related to the fracture processes at the source with the release-rebound processes associated with the molecular fields.

According to K.P. Teisseyre (private communication 2012) we may relate this motion to a possible vortex structure in solids, defined as the molecular vorticity:

$$
\varsigma_{k}=\varepsilon_{k p i} \frac{\partial v_{i}}{\partial x_{p}} \rightarrow \Xi_{k}=\varepsilon_{k p i} \frac{\partial w_{i}}{\partial x_{p}}
$$

where for the radial propagation we have:

$$
\Xi_{R}=\frac{\partial w_{z}}{R \partial \varphi}-\frac{\partial w_{\varphi}}{\partial z}, \quad \Xi_{\varphi}=-\frac{\partial w_{z}}{\partial R}, \quad \Xi_{z}=\frac{\partial w_{\varphi}}{\partial R} .
$$

Such a molecular vorticity we may be related to a gradient of pressure appearing in the $P$-wave propagation 


$$
\varepsilon_{s p q} \frac{\partial}{\partial x_{p}} \Xi_{q}-\frac{\partial}{V^{P} \partial t} \frac{\partial p}{\partial x_{s}}=0
$$

For the radial wave propagation, under the conditions $\partial p / \partial \varphi=0$ and $\partial p / \partial z=0$, we obtain:

$$
\frac{\partial \Xi_{z}}{R \partial \varphi}-\frac{\partial \Xi_{\varphi}}{\partial z}-\frac{\partial}{V^{P} \partial t} \frac{\partial p}{\partial R}=0, \quad \frac{\partial}{\partial z} \Xi_{r}-\frac{\partial}{\partial R} \Xi_{z}=0, \quad \frac{\partial}{\partial R} \Xi_{\varphi}-\frac{\partial}{R \partial \varphi} \Xi_{R}=0
$$

A similar problem may be applied to the plane waves along the $Z$-axis:

$$
\varepsilon_{s p q} \frac{\partial}{\partial x_{p}} \Xi_{q}-\frac{\partial}{V^{P} \partial t} \frac{\partial p}{\partial x_{s}}=0, \quad \frac{\partial}{\partial r} \Xi_{\varphi}-\frac{\partial}{r \partial \varphi} \Xi_{r}-\frac{\partial}{V^{P} \partial t} \frac{\partial p}{\partial Z}=0
$$

and under the condition $\partial p / \partial r=0$ and $\partial p / \partial \varphi=0$ we obtain

$$
\frac{\partial}{r \partial \varphi} \Xi_{Z}-\frac{\partial}{\partial Z} \Xi_{\varphi}=0, \quad \frac{\partial}{\partial Z} \Xi_{r}-\frac{\partial}{\partial r} \Xi_{Z}=0
$$

In such a way, in a solid continuum there might appear the molecular rotations with the pressure propagation waves and related events. In this way, it is possible to explain an abundance of the rotation motions which occur together and just after the $P$-wave arrivals.

Our considerations indicate that in the presented system of the shear and rotation wave propagation we have the correlations regarding the space and time derivatives; an interaction between these fields could be direct or shifted by $\pi / 2$. We should note that the asymmetric continuum theory includes a transition to the states close to fracture processes and, even further, to the fluid state. These fracture processes confirm the wave synchronization inside the molecular fields and the invariant 4D release-rebound system.

\section{CONCLUSIONS}

The presented 4D space-time system has been presented in a form of the rotation and shear strain vectors; their combined propagation is explained by the release-rebound interaction processes. At a seismic source, the molecular transport motions become transformed creating the real transport displacements. However, we cannot directly describe a fracture process in the frame of a continuum theory; we may only state that such a fracture is caused by an interaction of the real shear and rotation strains and related molecular transport displacements. 
Following the author's theory, the molecular transport motions may exist in a whole space, although we do not assume the independent propagation of the molecular fields; thus, the presented considerations may help us to understand an influence of these molecular wave motions on the appearance of another fracture, even at a remote distance. Of course, any molecular propagation is only apparent: it follows from the real propagation of the strains in solids or real transport in fluids (see also Teisseyre 2013).

\section{References}

Eshelby, J.D., F.C. Frank, and F.R.N. Nabarro (1951), The equilibrium of linear arrays of dislocations, Philos. Mag. Ser. 7 42, 327, 351-364, DOI: 10.1080/ 14786445108561060.

Kossecka, E., and R. DeWitt (1977), Disclination kinematic, Arch. Mech. 29, 633651.

Majewski, E., and R. Teisseyre (2013), Thermodynamics with rotation motions: fragmentation and slip, Acta Geophys. 61, 2, 281-310, DOI: 10.2478/ s11600-012-0072-2.

Rybicki, K. (1986), Dislocations and their geophysical applications. In: R. Teisseyre (ed.), Continuum Theories in Solid Earth Physics, Elsevier-PWN, Warszawa, 18-186.

Teisseyre, R. (1985), Creep-flow and earthquake rebound: System of the internal stress evolution, Acta Geophys. Pol. 33, 1, 11-23.

Teisseyre, R. (2011), Why rotation seismology: Confrontation between classic and asymmetric theories, Bull. Seismol. Soc. Am. 101, 4, 1683-1691, DOI: $10.1785 / 0120100078$.

Teisseyre, R. (2013), Molecular transport in fracture processes, Acta Geophys. 61, 1, 18-25, DOI: 10.2478/s11600-012-0057-1.

Teisseyre, R., and M. Górski (2012), Induced strains and defect continuum theory: Internal reorganization of load, Acta Geophys. 60, 1, 24-42, DOI: 10.2478/s11600-011-0046-9.

Varotsos, P.A. (2007), Comparison of models that interconnect point defect parameters in solids with bulk properties, J. Appl. Phys. 101, 12, 123503, DOI: 10.1063/1.2745359. 